\title{
eJRIEPS
}

Ejournal de la recherche sur l'intervention en éducation physique et sport

Hors-série $\mathrm{N}^{\circ} 2$ | 2018

À propos de certaines bases théoriques et pratiques des sports collectifs

\section{Tactique, stratégie, combinaison, schéma et intelligence tactique}

Jean-Francis Gréhaigne

\section{OpenEdition \\ 1 Journals}

Édition électronique

URL : http://journals.openedition.org/ejrieps/512

DOI : 10.4000/ejrieps.512

ISSN : 2105-0821

Éditeur

ELLIADD

Référence électronique

Jean-Francis Gréhaigne, «Tactique, stratégie, combinaison, schéma et intelligence tactique », eJRIEPS [En ligne], Hors-série N 2 | 2018, mis en ligne le 01 juillet 2018, consulté le 01 août 2019. URL : http:// journals.openedition.org/ejrieps/512 ; DOI : 10.4000/ejrieps.512

La revue eJRIEPS est mise à disposition selon les termes de la Creative Commons Attribution 4.0 International License. 


\section{Tactique, stratégie, combinaison, schéma et intelligence tactique}

Une équipe de football est une entité organisée en vue de se mesurer à une autre équipe dans une logique d'affrontement : l'une veut gagner et l'autre ne pas perdre. Elle est composée d'un groupe de joueurs ou de joueuses poursuivant un objectif commun : le gain du match (Mérand, 1977). L'interactionisme des opposants suppose une nécessaire adaptabilité car ils sont tous attaquants quand leur équipe possède le ballon et tous défenseurs dans le cas contraire. L'équipe est structurée selon des rideaux (Deleplace, 1979) ou selon des lignes de forces latérales (Teissié, 1962) arrières, milieux de terrain, avants - organisées en fonction d'un plan de jeu. Les joueurs sont répartis en tenant compte notamment de leurs compétences tactiques et motrices pour occuper un poste lié à une fonction tactique dominante.

Pour répondre à ces caractéristiques, la didactique des jeux collectifs étudie chacune des étapes de l'acte d'apprentissage et met en évidence l'importance du rôle de l'enseignant ou de l'entraîneur comme médiateur entre le joueur et le savoir... La question du rapport au savoir ne peut pas être bien analysée si n'est pas posée la question de la valeur opératoire de ce savoir. Dans les sports d'équipe, l'intelligence tactique (Gréhaigne, 2014) implique une quantité phénoménale de décisions sous pression temporelle, le tout étant façonnées par un arrière-plan décisionnel (Mouchet, 2005). Ce dernier comprend des éléments comme le score, le temps restant, l'importance du match, la fatigue et les stratégies convenues. La rapidité et l'efficacité du processus décisionnel suggère une adaptation à un environnement dynamique dans lequel il y a peu de séparations entre perception, prise de décision et action. Il y a donc lieu de comprendre l'intérêt théorique et de caractériser l'utilité pratique du concept d'intelligence tactique dans des situations de jeu complexes, évolutives, incertaines. Même si nous mobilisons principalement des travaux développés par les théoriciens des jeux sportifs, l'usage de ce concept concerne différents domaines et dépasse le seul cadre des sports collectifs.

Chez les militaires, l'intelligence tactique est l'art et la science de déterminer ce que l'opposition fait ou pourrait faire pour empêcher l'accomplissement de votre tâche. Elle est utilisée pour appuyer la prise de décisions relatives à la planification de l'affrontement et son exécution afin d'anticiper ce qui peut arriver dans la bataille. A ce propos, Carnot (1810) formule des principes d'action : primauté de l'offensive, action par surprise, décision rapide et action de masse. Il faut attaquer toujours l'ennemi là où il est faible et avec une supériorité de forces telle que la victoire ne puisse jamais être douteuse. L'auteur différencie stratégie et tactique : la tactique est l'art d'organiser et de disposer ses forces pour gagner une bataille ; la stratégie est l'art de mobiliser ses forces, d'organiser et de disposer ses batailles pour gagner la guerre. Pour approfondir ces premiers éléments 
concernant tactique et stratégie, nous allons nous appuyer sur les écrits de deux stratèges militaires renommés Sun Tzu et Von Clausewitz (Gréhaigne, 2014).

Sun Tzu préconise, à la fois, une vision très idéaliste et une perspective rationnelle de la guerre, où le vainqueur peut être prédit sur la base d'un ensemble de critères. Clausewitz voit la guerre comme un événement incertain. Même si ces deux conceptions ont des perspectives différentes sur la guerre, elles sont d'accord sur les stratégies. Ces deux stratèges conviennent tous les deux que la guerre doit être courte, l'effort concentré, néanmoins avec quelques légères différences. Sun Tzu voit la guerre comme une opération à petite échelle, tandis que Clausewitz pense que c'est un acte global qui exige des efforts d'une nation tout entière. Sun Tzu préconise l'utilisation de la tromperie, de pièges, tandis que Clausewitz envisage de combattre l'ennemi dans toute son entité. En fin de compte, c'est leur point de vue sur le hasard qui les sépare le mieux. Clausewitz voit la guerre comme un événement imprévisible qui est étroitement lié à la chance. Sun Tzu soutient quant à lui que le hasard ne joue pas un rôle dans la guerre et que le vainqueur peut être prédit sur la base d'un certain nombre de caractéristiques des deux belligérants.

Bien sûr, les sports collectifs ne sont pas la guerre. Mais il n'empêche que nous pouvons tirer des enseignements importants de ces deux auteurs concernant les jeux collectifs interpénétrés et de signes contraires (Jeu, 1977). Globalement le «plan de jeu type Von Clausewitz » vise à imposer son jeu et maîtriser totalement l'adversaire. Il s'agit pour l'entraîneur de dresser une suite d'actions idéales sous forme de schéma ou d'un plan de jeu contraignant. Ensuite, toutes les actions ou interventions consistent à appliquer ou à revenir aux solutions retenues. Quant à un «plan de jeu de type Sun Tzu», il laisserait venir l'adversaire pour mieux le surprendre, économisant ainsi ses forces pour la suite des opérations. Plutôt que de dresser un modèle qui serve de norme à son action, les joueurs devraient concentrer leur attention sur le cours du jeu, tel qu'ils s'y trouvent engagés, pour en déceler la cohérence et profiler son évolution. Il faut s'attacher à détecter les facteurs favorables dans la configuration du jeu en un mot s'appuyer sur le potentiel de la situation (Jullien, 1996).

\section{Quels enseignements pour les sports collectifs ?}

Évidemment dans les jeux, le dénouement ne vise pas une réelle destruction de l'équipe adverse mais une victoire sur des adversaires qui sont constitutifs de l'activité ludique. Ici, la compétition, l'émulation entre les joueurs représentent un moyen de se comparer à autrui, d'échanger avec lui et, ainsi, sources de progrès en ce qu'elles permettent à chacun de se situer dans une approche comparative à l'autre. Elles sont à la fois repère et dialogue : repère en ce qu'elles nous situent maintenant par rapport à celui ou ceux que j'ai choisi comme éléments comparateurs, et dialogue en 
ce qu'elles nous amènent à se comparer avec d'autres joueurs pour déterminer les progrès faits ou à faire.

Mais alors quels enseignements doivent être tirés des deux stratèges présentés dans le premier paragraphe ? Dans les jeux et les sports collectifs, la tactique constitue bien un concept polysémique qui se prête à beaucoup de commentaires. Souvent afin de simplifier, on distingue ce qui relève de l'organisation du jeu dans une l'équipe ou «plan de jeu » de ce que l'on peut appeler «intelligence tactique » ou «culture tactique ». Comme nous l'avons déjà mentionné, Gréhaigne (1989) définit l'organisation du jeu d'une équipe comme le résultat de l'interaction de plusieurs facteurs : les positions à occuper en fonction des consignes particulières ; l'ordre général, c'est-à-dire la forme d'ordre extérieur qui résulte des choix stratégiques généraux de l'équipe ; les positions produites par l'influence de l'adversaire avec adaptation ou non de l'équipe et des joueurs aux conditions de l'affrontement et enfin le joueur avec ses ressources, son statut, son expérience, son potentiel athlétique ...

Nous utilisons couramment comme définition pour la notion de système de jeu, celle proposée par Teodorescu, (1965) : «la forme générale d'organisation des actions offensives ou défensives des joueurs, par l'établissement d'un dispositif précis, de certaines tâches (postes et occupations du terrain) ainsi que de certains principes de collaboration entre ceux-ci» (p. 3). Une définition plus récente (Winkler, 1988) permet de la compléter ainsi : "le système de jeu représente une forme d'ordre extérieur où l'on distingue des groupes en différentes positions et où l'on détermine le nombre de joueurs assignés à ces groupes. On attribue à ces groupes certaines parties du terrain et certaines tâches dans le match. A l'intérieur de ces groupes on différencie de nouveau, pour assigner à chaque joueur une zone de terrain et des tâches » (p. 369).

A partir de ces données, il est clair que dans un match de sport collectif, les deux équipes représentent le plus souvent deux sous-systèmes non uniformes en interaction. Au plan collectif, les aspects suivants interviennent en priorité (Gréhaigne, Godbout \& Bouthier, 1999).

- Les décisions collectives évoquent l'ensemble des plans, principes ou directives d'action, retenus avant un match pour organiser l'activité de l'équipe et des joueurs pendant la rencontre. La stratégie mise au point peut soit concerner les grandes options générales de jeu, soit spécifier l'intervention des joueurs pour différentes classes de situation de jeu. Ainsi, on considère :

- Le rapport de forces qui est constitué par les liens antagonistes existant entre plusieurs joueurs ou groupes de joueurs opposés du fait de l'application de certaines règles du jeu qui déterminent un mode d'interaction.

- Le réseau de compétences qui renvoie aux relations entre les joueurs à l'intérieur d'une même équipe. Il influence les conduites et les comportements qui peuvent être identifiés chez les joueurs en fonction de leur statut et fonctions dans l'équipe. Il peut devenir un réseau de rivalité ... 
- L'activité d'un joueur est également influencée par sa concentration et sa vigilance mentale et par son degré de maîtrise de soi face aux contraintes et aux plaisirs associés au jeu. Ceux-ci influencent la lucidité des choix, alors qu'ils peuvent en même temps interférer avec l'exécution. Avec Gréhaigne (1992), nous dirons que l'organisation du jeu d'une équipe concourt à une meilleure efficacité offensive et défensive, à la fois par l'organisation générale rationnelle, mais aussi par l'action instantanée voire intuitive que représente l'intelligence tactique. Cet ensemble comporte une part de subjectivité d'où la proposition d'utiliser la verbalisation sur les conceptions et le vécu de l'action, comme moyen complémentaire d'identification et de développement de ces compétences. On peut également évoquer l'intérêt de prendre en compte l'intersubjectivité entre les joueurs avec le souci d'optimiser l'efficience collective (débat d'idées).

\section{De la stratégie au schéma}

En football, l'évolution, tant stratégique, tactique, technique que physique, a imposé des modifications inéluctables dans l'organisation du jeu liée la plupart du temps à un renforcement des défenses. Pour autant, organisation de jeu ne veut pas dire rigidité car il faut y voir une double adaptation des joueurs à l'organisation et de celle-ci aux joueurs.

La stratégie est un plan général dans lequel sont combinées des actions distinctes de manière à atteindre I 'objectif visé. La stratégie porte sur la méthode à suivre en vue gagner dans un affrontement global. Dans le cadre de la stratégie apparaissent aussi des éléments en rapport avec l'organisation du jeu. La stratégie représente les éléments discutés à l'avance pour s'organiser en fonction des caractéristiques propres de l'équipe adverse et de ses individualités. Gréhaigne et Godbout (1999, p. 4) précisent que « la stratégie peut soit concerner les grandes options de jeu, soit spécifier l'intervention des joueurs pour différentes classes de situations de jeu ». Il s'agit d'une anticipation liée aux informations dont on dispose sur les adversaires. La stratégie correspond à l'orientation globale du plan d'action. Elle permet d'atteindre directement les objectifs et propose une visée à moyen long terme. La stratégie envisage les gains espérés en fonction des risques encourus et elle relève du raisonnement et de la planification. La stratégie collective est répartie dans l'action de chaque joueur et n'a de sens que dans ce partage. Elle serait ainsi distribuée dans l'action de chaque partenaire ce qui doit conduire chacun des joueurs à définir la manière dont il s'inscrit dans ce projet.

La tactique dans le jeu se construit au cours de l'action en modifiant, selon les enseignements des événements, la perception d'informations ou la conduite envisagée. La tactique suppose chez le sujet les capacités à utiliser pour l'action, les déterminants et les aléas extérieurs. On peut la définir comme la méthode d'action propre au sujet en situation de jeu où, pour remplir son rôle, le joueur s'efforce de subir au minimum les contraintes, les incertitudes et les hasards du jeu et d'utiliser au 
maximum les capacités, potentiels et compétences dont il dispose. Les décisions prises et les actions motrices produites en fonction des configurations du jeu permettent d'évaluer la pertinence des réponses du point de vue tactique. Avec Teodorescu (1965 au Colloque de Vichy), nous parlerons aussi de tactique individuelle : "à côté de la tactique collective, il y aussi une tactique individuelle, dénommée improprement par certains spécialistes « technique individuelle ». La technique sportive ne peut être qu'individuelle et elle a comme fondement la connaissance des procédés spécifiques de la manœuvre du ballon, ainsi que l'accomplissement de certains mouvements sans ballon ». Pour définir la tactique individuelle, nous dirons que celle-ci représente l'ensemble des actions individuelles utilisées consciemment par un joueur dans ses interactions avec ses partenaires et ses adversaires, tant en attaque qu'en défense, pour bien souligner la singularité du problème et le caractère indissociable des compétences motrices et de la pensée tactique pour résoudre un problème en jeu, le tout dans un temps contraint.

Si l'on revient au jeu, les aspects stratégiques trouvent à leur tour nécessité d'adaptation. Les changements d'organisation des adversaires, l'évolution du score, les changements de joueurs, les blessures ou les sanctions administratives impliquent des réajustements si l'on veut rester efficace.

Pour Teodorescu (1965; 2013), les combinaisons tactiques sont la coordination des actions individuelles de deux ou plusieurs joueurs -dans une certaine phase du jeu- au but de réaliser une tâche partielle (temporaire) du jeu. Par exemple, la plus simple combinaison d'attaque est la passe (le passage du ballon). La circulation tactique est appliquée de préférence dans l'attaque, servant comme phase de préparation collective de l'attaque finale. C'est une forme supérieure de schéma de jeu. La circulation tactique s'applique dans l'ensemble du système du jeu auquel participe un grand nombre de joueurs. La circulation tactique représente une suite d'actions individuelles et de combinaisons tactiques effectuées suivant un certain plan d'organisation (Gréhaigne, 2014).

Les schémas de jeu consistent à s'organiser dans le cadre d'une circulation préétablie de joueurs et du ballon à partir de situations spécifiques (le plus souvent, à partir des situations de jeu arrêtées). On les retrouve à la fois dans le jeu pour rendre les mouvements offensifs plus efficaces, mais aussi lors des phases statiques. Un «schéma tactique» (Teodorescu, ibid) constitue un programme préétabli qui propose une régulation automatique de façon à faire face de façon économique à des situations relativement stables. Ils permettent de surprendre l'adversaire à l'aide d'une avance initiale ou tout simplement en jouant plus vite que l'adversaire et de devancer le replacement défensif comme en volley-ball et au football américain. En football ou en rugby, excepté pour les phases statiques ou les lancements de jeu, le recours à des schémas de jeu pour assurer la continuité du jeu est rarement opportun. En effet, le désordre s'installe très rapidement avec les divers déplacements des joueurs et du ballon et vient contrecarrer le bel ordonnancement du schéma tactique. Dans ce cas, une adaptation tactique est nécessaire pour passer du schéma aux réalités de la configuration 
momentanée du jeu et de la circulation du ballon dans le rapport d'opposition en cours. Mais souvent, un usage mal compris de l'apprentissage de tels schémas stricts étouffe la personnalité du joueur, le réduit et le contraint à réaliser des actions individuelles trop précises, le "sclérose" et freine ses possibilités d'expression et de développement d'une pensée tactique.

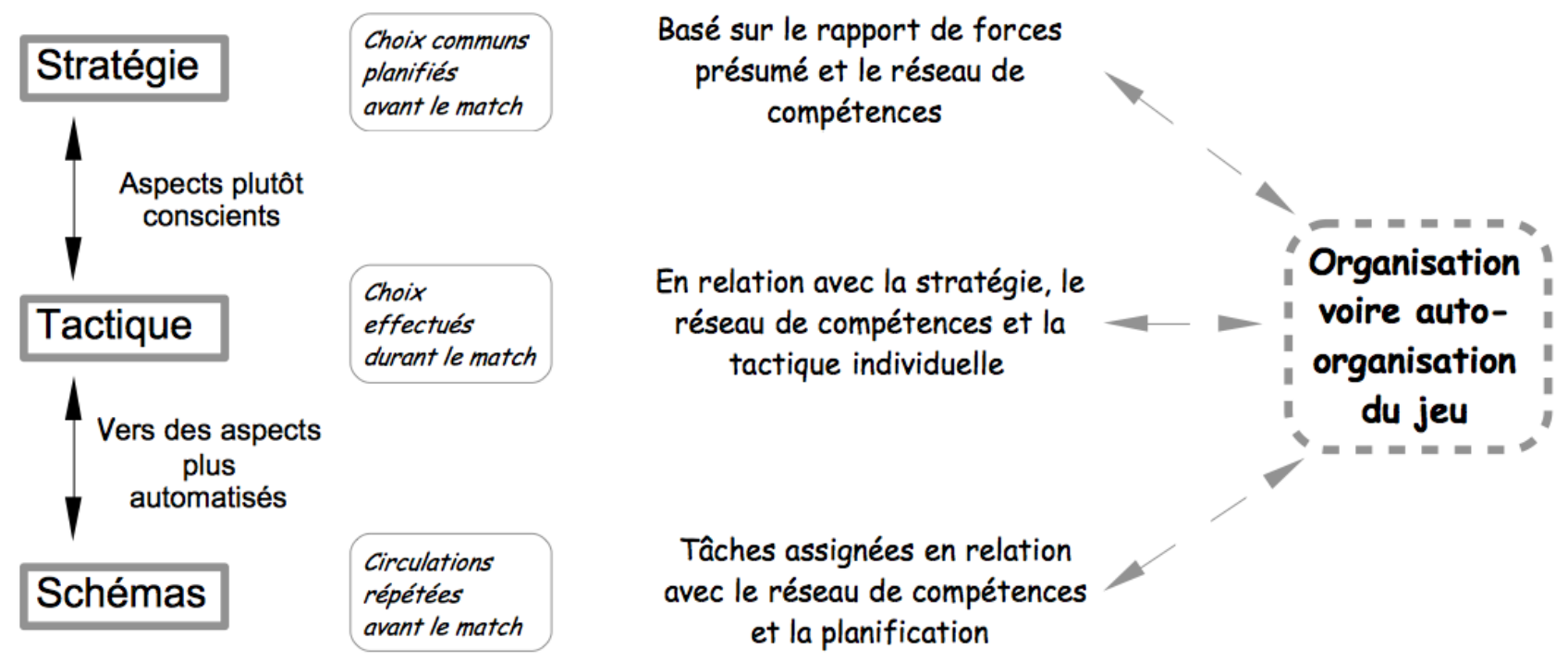

Figure 1. Quelques caractéristiques de stratégie, tactique et schémas de jeu (Gréhaigne, 2014)

En football, le fait de ne pas «tenir le ballon » a pour conséquence une centration plus importante sur la circulation tactique en raison des difficultés à conserver la balle. Dans les sports collectifs où l'on manie la balle facilement, le problème est un peu différent. En effet, les progrès effectués permettent assez rapidement de passer d'un jeu de transition d'un but à l'autre à un jeu sur espace stabilisé. Ce progrès pose des problèmes dont la résolution passe en partie par une circulation des joueurs connue et identifiée par l'équipe.

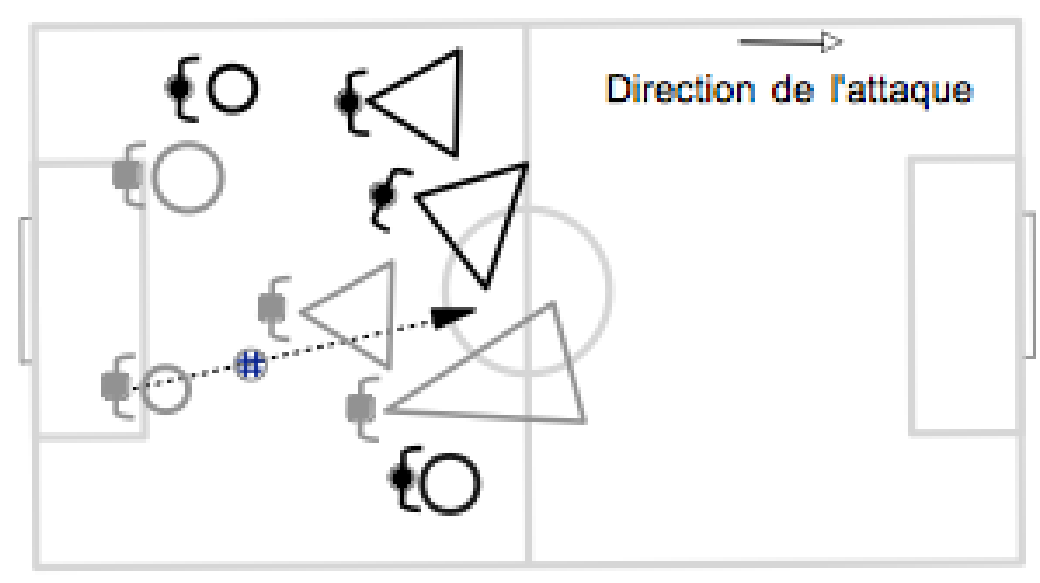

Figure 2. Configuration dynamique du jeu de T0 à $\mathrm{T}+1$ 
Le ballon et les joueurs circulent successivement vers des endroits du terrain dont les grandes lignes sont établies à l'avance. Cette circulation tactique est élastique et suppose la détermination non seulement de certains principes de déplacement de circulation du ballon et des joueurs, mais aussi du sens et du rythme de ces différents mouvements. En outre, avant de passer le ballon le porteur de balle devra avoir une idée de la position approximative du futur réceptionneur.

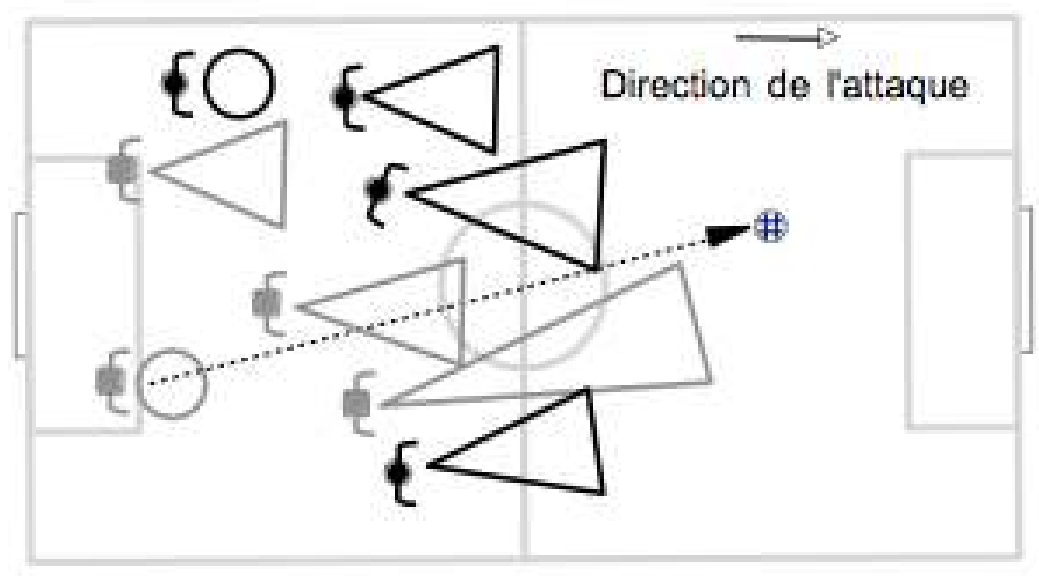

Figure 3. Configuration dynamique du jeu de $\mathrm{T} 0$ à $\mathrm{T}+2 \mathrm{~s}$

En effet, dans le jeu, en une seconde la distance parcourue par un ou plusieurs joueurs en fonction de leur vitesse ou de leur accélération peut être importante. La représentation spatiale de ces phénomènes est illustrée dans les figures 2 et 3.

La modélisation du jeu en mouvement avec les notions de secteurs d'action pour les attaquants et d'intervention pour les défenseurs (Gréhaigne \& Bouthier, 1994 ; Gréhaigne, Bouthier \& David, 1997) a permis de mieux comprendre l'importance des rapports de vitesse entre les joueurs pour faire évoluer le rapport de forces. Une configuration dynamique du jeu est établie à l'aide de la distribution des joueurs et du ballon sur le champ de jeu en fonction de leurs positions, de leurs orientations et de leurs vitesses de déplacement dans un intervalle de temps donné.

Il est évident qu'en deux secondes, avec des joueurs en déplacement, la séquence de jeu a beaucoup évolué. Certains joueurs ont peu bougé mais d'autres, lancés à pleine vitesse, ont donné une expansion considérable à cette configuration dynamique. La caractéristique de la circulation des joueurs est qu'elle peut être à la fois très rapide mais aussi entrecoupée d'une succession d'arrêts. Alors, les transitions du jeu entre deux états (deux configurations momentanées du jeu) deviennent des éléments d'observation essentiels pour des décisions appropriées. Barrage / poursuite, avance / retard deviennent des éléments premiers de la prise d'informations. 


\section{L'expérience du joueur}

Dans une perspective constructiviste, lorsqu'un joueur intériorise un savoir, on dira qu'il construit cette connaissance, qui lui appartient alors en propre, car le même savoir construit par un autre joueur ne sera jamais tout à fait le même. La question de l'acquisition et du développement de ces savoirs d'actions devient alors essentielle. Savoyant (2008) note que «la question de la formalisation des savoirs implicites comporte un paradoxe, sinon une contradiction, puisqu'il s'agit de formaliser et donc d'expliciter, des savoirs dont on a dit qu'ils étaient issus d'abord de l'activité elle-même et qu'il était difficile, sinon impossible de les expliciter, de les énoncer» (p.2). Pour résoudre ce paradoxe, il faut envisager l'idée que les savoirs non conscients se transmettent implicitement dans le cadre général de l'apprentissage par l'action. La pression temporelle dans les sports collectifs vient sans doute ici apporter des contraintes supplémentaires. Ceci étant posé, nous allons maintenant nous intéresser aux problèmes spécifiques posés par la formation tactique des joueurs.

Considérer la tactique

Notre approche consiste à subordonner le travail technique, physique et psychologique au travail de la tactique collective. Le référentiel commun paraît aussi constituer l'interface entre l'intelligence tactique des joueurs en situation et les stratégies, le plan de jeu, les schémas tactiques.

Cette approche a le mérite de présenter un modèle d'apprentissage qui vise à développer l'intelligence de jeu des joueurs, car basée sur une méthodique acquisition des principes de jeu offensifs et défensifs dans un contexte d'opposition. Trois moments principaux constituent l'essentiel des problèmes tactiques du football que doivent résoudre les joueurs individuellement et / ou collectivement. Chaque problème est posé en fonction des rapports d'opposition instantanés qui peuvent être favorables à l'attaque, favorables à la défense ou équilibré et en devenir (Tableau I). Dans le devenir, les transitions entre deux états sont toujours porteuses d'informations notables sur l'évolution du jeu comme les lieux de récupération du ballon (Duprat, 2005).

La volonté principale de l'apprentissage et du perfectionnement à partir des principes de jeu est bien de permettre d'établir un cadre collectif commun aux joueurs et joueuses quel que soit la forme de jeu proposée. En effet, une des dérives collectives a pour conséquence une récitation de formes de jeu peu adaptée aux rapports d'opposition instantanés. Notre ambition est de proposer un fil conducteur commun que les joueurs s'approprient afin de jouer les formes de jeu collectives basées sur des principes de jeu communs. 
Tableau I. Différents états des rapports d'opposition.

\begin{tabular}{|l|l|l|}
\hline Les rapports d'opposition sont & \multicolumn{1}{|c|}{ Organisation de l'attaque } & Organisation de la défense \\
\hline Favorables à l'attaque & $\begin{array}{l}\text { Contre-attaque, attaque rapide } \\
\text { - -ller vite, jeu direct } \\
\text { - conserver son avance sur le } \\
\text { replacement défensif }\end{array}$ & $\begin{array}{l}\text { Défense à la poursuite } \\
\text { - retarder l'action offensive } \\
\text { - protéger l'accès au but } \\
\text {-reconstruire un barrage }\end{array}$ \\
\hline Equilibrés et en devenir & $\begin{array}{l}\text { Attaque placée } \\
\text { - créer le surnombre } \\
\text { - jouer en mouvement } \\
\text { - créer de l'incertitude }\end{array}$ & $\begin{array}{l}\text { Défense en barrage } \\
\text { - rester devant le porteur } \\
\text { créer une zone temporaire } \\
\text { côté ballon }\end{array}$ \\
\hline Favorables à la défense & $\begin{array}{l}\text { Attaque placée } \\
\text { - conserver le ballon et pro- } \\
\text { gresser } \\
\text { - contourner, prendre à revers }\end{array}$ & $\begin{array}{l}\text { Défense en barrage } \\
\text { ballon } \\
\text { - conserver la stabilité }\end{array}$ \\
\hline
\end{tabular}

L'idée est bien de ne pas stéréotyper les réponses tactiques, mais de permettre une lecture appropriée du jeu reliée aux principes de jeu afin de garantir une continuité dans le jeu. En outre, le développement des principes de jeu au sein d'une circulation tactique permet de construire un sens tactique lié au perfectionnement technique car il est plus aisé de travailler la tactique individuelle autour d'un fil conducteur commun que de modéliser chaque forme de jeu pour y apporter des réponses différentes.

Après avoir défini les aspects stratégiques et tactiques dans lequel évolue le football, il est maintenant temps de voir quelques règles d'action et principes de jeu dont devraient disposer les joueurs afin de résoudre les problèmes qui se posent à eux durant un match.

L'apprentissage des aspects tactiques est nécessaire car ne pas poser le problème de la formation de la pensée tactique, c'est amener les élèves les joueurs à apprendre schématiquement et donc à jouer de même. En outre, il est bien connu que l'exécution, versant moteur d'un choix tactique, coûte de l'énergie. En effet, la fatigue affecte non seulement la qualité d'exécution des compétences motrices, mais affecte aussi la lucidité des choix. 
Tableau II. Quelques règles d'action et principes de jeu en fonction du jeu.

\begin{tabular}{|c|c|}
\hline Conserver la balle & Récupérer le ballon \\
\hline $\begin{array}{l}\text { Utilisation, création d'espaces libres } \\
\text { - Occupation large et profonde du terrain } \\
\text { - Déplacements et jeu dans les intervalles } \\
\text { Organisation à double effet } \\
\text { - augmentation des possibilités d'échanges en } \\
\text { utilisant les appuis et les soutiens }\end{array}$ & $\begin{array}{l}\text { Réduction d'espaces et de mouvement } \\
\text { - Pression sur le porteur du ballon } \\
\text { - Repli défensif vers son but à l'intérieur du } \\
\text { terrain } \\
\text { - Marquage des receveurs potentiels }\end{array}$ \\
\hline Jeu de transition & Jeu de transition \\
\hline $\begin{array}{l}\text { Utilisation, création d'espaces libres } \\
\text { - Fixation latérale puis renversement } \\
\text { - Fixation axiale puis débordement } \\
\text { - Coordination appui, soutien, appels } \\
\text { - Dédoublements, écrans } \\
\text { Utilisation du jeu en mouvement } \\
\text { - Limitation du nombre d'échanges } \\
\text { - Alternative jeu direct - indirect } \\
\text { - Changement de rythme avec et sans le ballon } \\
\text { - Surnombre offensif local }\end{array}$ & $\begin{array}{l}\text { Réduction d'espaces et du mouvement } \\
\text { - Augmentation de la densité côté ballon et dans } \\
\text { l'axe du but } \\
\text { - Pression sur le porteur de balle, marquage des } \\
\text { receveurs potentiels } \\
\text { - S'organisation en lignes de forces } \\
\text { - Suppléance entre les lignes } \\
\text { - Surnombre défensif localisé } \\
\text { - Placements pour intercepter } \\
\text { - Actions défensives au sein d'un plan de jeu }\end{array}$ \\
\hline Marquer & Défendre la cible \\
\hline $\begin{array}{l}\text { Utilisation du jeu en mouvement } \\
\text { - Limiter le nombre de touches de balle } \\
\text { - Réception en mouvement ou en l'air } \\
\text { - Surnombre offensif } \\
\text { - Utilisation de la feinte }\end{array}$ & $\begin{array}{l}\text { Réduction de l'espace et du mouvement } \\
\text { - Couverture, renforcement de l'axe du but } \\
\text { - Recul frein sans se faire consommer } \\
\text { - Rester en barrage } \\
\text { - Anticipation des actions offensives adverses }\end{array}$ \\
\hline
\end{tabular}

C'est une idée aujourd'hui largement admise qu'il n'y a pas que des connaissances explicites à propos du jeu et que les sports collectifs ne se réduisent pas qu'à l'application de quelques règles ou procédures fondées sur de seuls savoirs théoriques, scientifiques et technologiques (Mouchet, 2005). Dans la mise en œuvre de leurs activités, les joueurs élaborent et utilisent d'autres savoirs que l'on qualifiera de savoirs d'action, savoirs d'expérience, savoirs implicites, savoirs tacites, savoirs informels, etc. Au-delà de la diversité des catégories, ces savoirs présentent trois caractéristiques :

- ils se construisent et se développent souvent dans l'exercice même de l'activité ;

- ils sont difficiles à expliciter et à énoncer systématiquement ;

- c'est l'expérience qui fait d'abord la différence entre débrouillés et joueurs expérimentés.

Dans une perspective constructiviste, lorsqu'un joueur intériorise un savoir, on dira qu'il construit cette connaissance, qui lui appartient alors en propre, car le même savoir construit par un autre joueur n'en sera jamais tout à fait le même. 


\section{Conclusion}

Pendant longtemps, la culture tactique d'un joueur a été considérée comme un don et de ce fait difficilement transmissible par l'apprentissage. Heureusement, on est revenu de cette conception et la formation tactique devient un enjeu fondamental dans la culture d'un joueur. Dans la vie courante, on oppose trop rapidement une formation scolarisée et méthodique à l'apprentissage sur le tas. Il y a de bonnes raisons à cela, mais il y en a de mauvaises : d'une part, l'école ou le club offre des opportunités d'apprentissage, y compris des situations, que n'offre pas la vie quotidienne extrascolaire. D'autre part, l'analyse des activités hors école montre que les hommes et les femmes ne tirent pas forcément un égal profit de l'expérience. Cette inégalité résulte pour une part non négligeable des différences entre individus dans leur rapport au savoir. Le joueur doit donc prendre appui sur le potentiel inscrit dans la configuration du jeu pour prendre une « cascade de décisions ». Ainsi se trouve exclue l'idée de prédéterminer le cours des évènements en fonction d'un plan rigide qu'on aurait dressé par avance. C'est le passage de l'évaluation des forces en présence au potentiel d'actions qui s'en dégage qui représente un moment clé de la réflexion. En dernier ressort, un joueur accroît ses compétences quand il dispose d'un éventail de ressources, toutes disponibles et stabilisées, pour faire face à une grande variété de situations qu'il sera amené à rencontrer.

\section{Bibliographie}

Carnot, L. (1810). De la défense des places fortes. Paris : Courcier.

Deleplace, R. (1979) Rugby de mouvement-Rugby total. Paris : E.P.S.

Duprat, E. (2005). Approche technologique de la récupération du ballon lors de la phase défensive en football, contribution à l'élaboration de contenus de formations innovants. Thèse de Doctorat (non publiée). École Normale Supérieure de Cachan.

Gréhaigne, J.F. (1989). Football de mouvement. Vers une approche systémique du jeu. Thèse (non publiée). Université de Bourgogne.

Gréhaigne, J.F. (1992). L'organisation du jeu en football. Paris : ACTIO.

Gréhaigne, J.F. (2014). L'intelligence tactique : quelques données. In Gréhaigne, J.-F. (Ed.). L'intelligence tactique. Des perceptions aux décisions tactiques dans les sports collectifs (pp. 25-40). Besançon : Presses de 1’Université de Franche-Comté.

Gréhaigne, J.F., \& Bouthier, D. (1994). Analyse des évolutions entre deux configurations du jeu en football. Science et Motricité, 24, 44-52. 
Gréhaigne, J.F., \& Godbout, P. (1999). La prise de décision de l'élève en sport collectif. Communication orale au Colloque AIESEP, Qu'apprennent les élèves en faisant des activités physiques et sportives ? Besançon, France.

Gréhaigne, J.F., Godbout, P., \& Bouthier, D. (1999). The foundations of tactics and strategy in team sports. Journal of Teaching in Physical Education, 18, 159-174.

Gréhaigne, J.-F. , Bouthier, D., \& David, B. (1997). Dynamic systems analysis of the opponent relationships in the collective actions in soccer. Journal of Sports Sciences, 15, 137-149.

Jeu, B. (1977). Le sport, l'émotion, l'espace. Paris : Vigot.

Jullien, F. (1996). Traité de l'efficacité. Paris : Grasset

Mouchet, A., (2005). Modélisation de la complexité des décisions tactiques en rugby, eJRIEPS, 7 , 3-19.

Mérand, R. (1977). Considérations sur une problématique de rénovation des contenus de l'Education Physique en rapport avec les activités sportives contemporaines. In CPS de la FSGT (Ed.) L'éducateur face à la haute performance olympique (pp.05-27). Paris : Sport et Plein air.

Teodorescu, L. (1965). Principes pour l'étude de la tactique commune aux jeux sportifs collectifs. Revue de la S.I.E.P.E.P.S., 3, 29-40. (texte publié à nouveau en 2013 dans la revue eJRIEPS, 28, 99-117).

Savoyant, A. (2008). Quelques réflexions sur les savoirs implicites [A few thoughts on implicit knowledge]. Travail et Apprentissages, 1, 92-100.

Sun Tzu (1972). L'art de la guerre. Paris : Flammarion (Traduit du chinois vers l'anglais par Samuel Griffith en 1963), puis traduit de l'anglais vers le français par Francis Wang en1972).

Teissié, J. (1962). Le Football. Paris, E.P.S.

Von Clausewitz, C. (1989). De la guerre. Paris : Lebovici (traduction d'un livre publié originellement en Allemagne en 1832).

Wallon, H. (1941). L'évolution psychologique de l'enfant. Paris : Armand Colin.

Winkler, W. (1988). A new approach to the video analysis of tactical aspects of soccer. In T. Reilly, A. Lees, K. Davis \& W.-J. Murphy (Eds.) Science and Football I. Londres : E. \& F.N. SPON, 368-372. 